\title{
ANÁLISIS DE LA POLÍTICA PARA EL CONTROL DE LA TUBERCULOSIS EN COLOMBIA
}

\author{
Dilia Fontalvo-Rivera',a, Doris Gómez-Camargo ${ }^{1, b}$, Rubén Gómez-Arias²,c
}

\begin{abstract}
RESUMEN
Se pretende analizar la política para el control de la tuberculosis en Colombia entre 1975 y 2010. Durante los años 80 se observó un esfuerzo en la gestión de los programas de control que se reflejó en la reducción de la incidencia y en mortalidad. A partir de 1993, cuando se desmontó el Sistema Nacional de Salud y se privatizó la gestión de los recursos, los indicadores de desempeño del programa de control mostraron un deterioro importante. A partir de 2009 se observa un esfuerzo por precisar y fortalecer la política de control de la tuberculosis; sin embargo, no tocan la estructura del modelo de salud y los resultados encontrados no muestran aún una recuperación importante que permita lograr para el 2015 un aumento en la notificación, curación de al menos el $85 \%$ de pacientes con tuberculosis y la reducción en un $50 \%$ de la incidencia y mortalidad.
\end{abstract}

Palabras clave: Tuberculosis; Políticas; Salud pública; epidemiología; Colombia (fuente: DeCS BIREME).

\section{ANALYSIS OF THE POLICY FOR TUBERCULOSIS CONTROL IN COLOMBIA}

\begin{abstract}
The article aims to analyze the policies for the control of tuberculosis in Colombia between 1975 and 2010. During the 1980 s, there was an effort in management of the control programs that was reflected in the reduction of incidence and mortality. Since 1993, when the National Health System was dismantled and resource management was privatized, the performance indicators of the control program showed significant deterioration. Since 2009 there has been an effort to clarify and strengthen the control policy of tuberculosis. However, the structure of the health model remains untouched and results don't show significant recovery that would allow by 2015 an increase in the notification and cure of at least $85 \%$ of patients with tuberculosis, and a reduction in incidence and mortality of $50 \%$.
\end{abstract}

Key words: Tuberculosis; Policy; Public health; Epidemiology; Colombia (source: MeSH, NLM).

\section{INTRODUCCIÓN}

La morbilidad y mortalidad por tuberculosis (TB) se han relacionado con múltiples determinantes biológicos, ambientales, económicos y culturales, y su control demanda acciones complejas de tipo social, preventivo y terapéutico. Desde 1982 McKeown (1) demostró que la TB venía desapareciendo en Europa mucho antes del descubrimiento de la quimioterapia, sugiriendo que su control depende en gran medida de condiciones económicas y sociales. La prevención de la infección y la enfermedad se apoya en el mejoramiento de las condiciones materiales de vida, la vacunación con BCG, el estudio y protección de contactos domiciliarios de los enfermos y el uso de quimioprofilaxis para contactos sanos, en casos indicados. El manejo médico incluye el estudio de sintomáticos respiratorios (SR), la detección precoz, la quimioterapia, el seguimiento estrecho de los enfermos, la vigilancia de resistencia al tratamiento y el control de recaídas ${ }^{(2)}$.

A pesar de los esfuerzos, la TB sigue siendo un problema de salud pública, y es después del sida, la segunda causa mundial de mortalidad causada por un agente infeccioso. En 2011, en las Américas, los casos se estimaban en 268000 y las defunciones en

\footnotetext{
Grupo investigación UNIMOL, Universidad de Cartagena. Cartagena de Indias, Colombia.

Facultad Nacional de Salud Pública. Universidad de Antioquia. Antioquia, Colombia.

Médico pediatra; ${ }^{\mathrm{b}}$ doctora en Biología Molecular; ${ }^{\mathrm{c}}$ doctor en Salud Pública.

Recibido: : 05-11-14 Aprobado: 02-04-14
}

Citar como: Fontalvo-Rivera D, Gómez-Camargo D, Gómez-Arias R. Análisis de la política para el control de la tuberculosis en Colombia. Rev Peru Med Exp Salud Publica. 2014;31(4):775-80. 
30 000. Diez países concentraron el $80 \%$ de los casos de TB en todas sus formas y de ellos Brasil, Perú y Haití reportaban el $52 \%$ de los casos. Después de África, las Américas reportan el mayor número de casos de coinfección TB/VIH con un estimado de 35 mil casos, de los cuales 8100 eran TB multidrogorresistente (TBMDR) y TB extensamente resistente (TB-XDR).

El presente artículo pretende analizar la política para el control de la TB aplicada en Colombia entre 1975 y 2010, con base en fuentes secundarias, para la cual se hizo una revisión narrativa no sistemática de la literatura disponible relacionada con el control de la TB en Colombia.

\section{POLÍTICA DE CONTROL DE LA TUBERCULOSIS EN COLOMBIA}

Entre 1975 y 1993, operó en Colombia un Sistema Nacional de Salud (SNS) financiado por la vía de impuestos y rentas estatales, controlado por el Ministerio de salud. EL SNS desarrollaba dos acciones específicas para el control de la TB, ofrecidas gratuitamente por las instituciones estatales de salud: la vacunación de todo recién nacido con la BCG, y el programa Control de TBC dirigido verticalmente desde el Ministerio. Dicho programa consistía en el tamizaje obligatorio de todo SR con baciloscopías seriadas, la captación de los enfermos y el suministro de un esquema terapéutico, idealmente supervisado, para asegurar la adherencia y la curación, y evitar la resistencia durante los tres a nueve meses que duraba el tratamiento.

En 1987 aparecieron los primeros casos de $\mathrm{VIH} / \mathrm{Sida}$ en Colombia y la TB extrapulmonar se incluyó como un evento de vigilancia obligatoria. En 1990 la Ley 10, acogiendo las directrices del Banco Mundial, inició el desmonte del SNS. Este proceso se consolidó en 1993, cuando la ley 100 organizó los servicios de salud con criterios de mercado asignado su administración a aseguradoras privadas que controlaban los recursos ${ }^{(4,5)}$. Esta reforma segmentó la población en tres grupos con base en su capacidad de contribuir a la financiación del Sistema General de Seguridad Social en Salud (SGSSS): los cotizantes y sus familias (régimen contributivo), los afiliados cuyo aseguramiento era financiado por el estado (régimen subsidiado) y la población sin recursos que debía ser atendida en las instituciones estatales (régimen vinculado) ${ }^{(4-7)}$.

A partir de entonces, el control de la TB pasó a formar parte del paquete de beneficios que las aseguradoras ofrecen a sus afiliados, mientras la población no asegurada debe consultar a los hospitales estatales. La
Ley 100 de 1993 entró en vigencia en enero de 1995, sin embargo, las acciones de control de la TB estuvieron en un limbo administrativo hasta 1998, cuando el Ministerio definió un paquete de salud pública denominado Plan de Atención Básica a cargo de las secretarías municipales de salud, el cual incluía acciones colectivas para prevenir la TB, mientras el tratamiento individual de los enfermos se asignó a las aseguradoras. Sin embargo, el programa de control no disponía de formularios oficiales para el control y registro de la TB hasta 2000, cuando la Resolución 412 del Ministerio de Salud reglamentó los procedimientos e intervenciones relacionadas con la atención de enfermedades de interés en salud.

Algunos autores destacan el deterioro de la salud pública a partir de la Ley 100 de $1993^{(4,5)}$. En relación con la TB, Ayala y Kroeger ${ }^{(3)}$ describieron el fraccionamiento del programa de control, de los recursos y de las actividades entre tres tipos de actores: los entes territoriales, las entidades aseguradoras (EPS), y las instituciones prestadoras de servicios de salud IPS; su estudio concluyó que el afán de aumentar la rentabilidad financiera, la falta de interés en el programa, la negligencia y la descoordinación de los actores, obstaculizaron la búsqueda de SR y de estudio de contactos, redujeron el número de pacientes en tratamiento, y deterioraron la calidad y eficiencia de las acciones de control.

En 2000, y en respuesta a las críticas que recibía el modelo de salud, el Ministerio promulgó la resolución 412 donde adoptó guías de atención para enfermedades de interés en salud pública incluyendo la TB ${ }^{(8-11)}$.

A partir de entonces se produjeron algunas disposiciones que reglamentan la salud pública; en 2006 el Decreto 3518 creó y reglamentó el sistema de vigilancia en salud pública, y el Decreto 2323 reguló parcialmente la Ley 9 de 1979 en relación con la Red Nacional de Laboratorios asignando al INS el monitoreo de la Red Nacional de Laboratorios en el diagnóstico de la TB. Un año más tarde la Ley 1122 de 2007 modificó el concepto de Plan de Atención Básica por el Plan Nacional de Salud Pública de intervenciones colectivas (PIC); en el marco de esta norma, el Decreto 3039 de 2007 adoptó el Plan Nacional de Salud Pública 2007-2010 y el Plan Estratégico Colombia libre de TB (PECT) 2006-2015: Alto a la TB, reglamentado por el Ministerio (10-14).

\section{PLAN ESTRATÉGICO COLOMBIA LIBRE DE TUBERCULOSIS 2006-2015}

Este Plan define la justificación del programa de control de la TB y alude al compromiso que Colombia hizo ante el Consejo Nacional de Política Económica y Social (CONPES) para el logro de los Objetivos del 
Desarrollo del Milenio, entre ellos, considerar la TB como una prioridad y expandir y adaptar la estrategia de terapia acortada altamente supervisada (TAES); define también el problema de la alta tasa de incidencia y la baja detección de los SR ${ }^{(15-19)}$. Entre los ocho objetivos de esta norma técnica se pretende garantizar el acceso a servicios de detección temprana, diagnóstico y tratamiento de las personas con TB. A este respecto se fijan varias metas que deben cumplirse en 2015, entre ellas: captar al menos el $80 \%$ de SR y cubrir con TAES al menos el $85 \%$; curar más del $85 \%$ de los casos de TB pulmonar con baciloscopia positiva; notificar más del $70 \%$ de los casos nuevos con baciloscopia positiva; reducir la incidencia de TB y disminuir la mortalidad y la prevalencia en un $50 \%$ respecto a 1990 . Aunque estas disposiciones promueven criterios y requisitos de tipo técnico, no desarrollan mecanismos de sanción en casos de incumplimiento.

Uno de los objetivos del Plan es mejorar la calidad y oportunidad para el diagnóstico y seguimiento bacteriológico a través de la Red Nacional de Laboratorios, mediante la ampliación al 100\% de la cobertura y control por cultivo, la asistencia técnica y monitoreo de los laboratorios, la capacitación a cargo del INS, la evaluación externa del desempeño de la baciloscopia y la cobertura al $100 \%$ en la vigilancia de la resistencia de los fármacos antituberculosos. Aunque la concentración de baciloscopia, que es un indicador que determina la sensibilidad diagnóstica de la primera, segunda y tercera baciloscopia, se ha superado a partir de 1996, sigue estando inferior a 2,5, que es la meta propuesta en el PECT y es atribuido a que muchos SR solo son examinados con la primera muestra; perdiéndose la oportunidad de diagnosticar del 15 al $30 \%$ y del 5 al $10 \%$ de los casos bacilíferos en la segunda y tercera toma respectivamente ${ }^{(15,16)}$.

El Plan Estratégico promueve también el fortalecimiento del sistema de vigilancia de la TB integrado al Sistema de Vigilancia en Salud Pública (SIVIGILA) en lo que se refiere a la investigación epidemiológica de campo y estudios de contacto. Esta disposición puede ser difícil de aplicar en poblaciones rurales y suburbanas que identifiquen adecuadamente los casos sospechosos en las poblaciones más susceptibles; allí, las personas consideradas de alto riesgo, carecen de servicios de salud y de red local de laboratorios que puedan detectar la micobacteria y mucho menos las cepas resistentes a antifímicos ${ }^{(20-22)}$. El objetivo 6 del Plan Estratégico promueve la participación comunitaria para la prevención y control de la TB; a este respecto, propone que el $50 \%$ de las entidades territoriales desarrollen estrategias de abogacía, comunicación y movilización social con la incorporación de grupos afectados en las actividades de control (19,23,24); aunque la educación y la movilización social se presentan como un lineamiento básico de la estrategia, no se encontraron referencias alusivas a la participación directa de un representante de la comunidad, que en casos de poblaciones susceptibles con un grado de costumbres y cultura particulares como los indígenas y convictos pueden requerir un consentimiento previo en la aplicación de los planes de acción.

Involucrados. Entre los actores involucrados en la elaboración de la política de control de TB se identificaron: el Ministerio de Salud, particularmente la Dirección de Salud Pública, la Coordinación de Promoción y Prevención y el Grupo Funcional Nacional de TB; la OMS/OPS y el INS. No se encontró referencia a la participación de representantes de la comunidad en el proceso. El monitoreo y la evaluación del PECT, quedó bajo responsabilidad del Ministerio de Salud y el INS. Los municipios son responsabilidad de las entidades gubernamentales departamentales y distritales ${ }^{(15,24)}$.

Financiación. Según la Ley de Presupuesto General, los medicamentos definidos en las guías de tratamiento de todos los casos reportados, deben adquirirse utilizando los recursos transferidos a los departamentos y distritos para la vigilancia, prevención y control de la TB. Entre 2002 y 2008 se asignaron US\$ 6809268 y se invirtieron en medicamentos US\$ 8215 688. Para 2009, la partida asignada por concepto de transferencias fue de US\$ 1568 534, US\$ 863005 para adquisición de medicamentos y US\$ 609027 para la ejecución del Plan Estratégico: "Colombia Libre de tuberculosis 2006-2015". En el 2012, el Ministerio de Salud con el Fondo Mundial de Lucha contra la TB, inició un proyecto a desarrollar en cinco años con una inversión de 9,5 millones de dólares para el control de la enfermedad (25).

Vigilancia. Las políticas vigentes definen la vigilancia y el monitoreo del programa; en la práctica, sin embargo, persisten limitaciones en la notificación, identificación de SR y estudio de contactos que, incluso, puede estar influyendo en la clasificación adecuada del riesgo epidemiológico de una región.

Indicadores de desempeño de la política. Según el PECT, la captación de SR debería corresponder al 10\% del total de consultas médicas de primera vez por todas las causas, registradas en el período inmediatamente anterior en la población mayor de 15 años, sin embargo, el subregistro de SR es alto, porque este dato no siempre es informado por los departamentos y distritos. Desde 1996 el porcentaje de captación de SR que era de $38,3 \%$ comenzó a disminuir y, en 1998 , se sitúo en $27,3 \%$ para iniciar un paulatino ascenso y colocarse en 


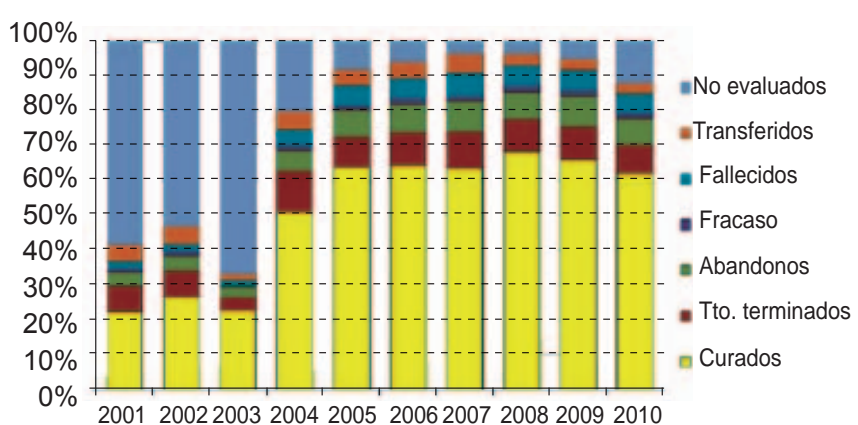

Figura 1. Resultados del tratamiento en pacientes con baciloscopía positiva, Colombia 2001-2010. Fuente: Programa Nacional de TB. Ministerio de salud República de Colombia.

el 2000 en $36,7 \%$. Sin embargo, este indicador aún se encuentra lejos del mínimo propuesto $(80 \%)^{(24-26)}$.

En el objetivo 1 del PECT se propuso curar más del $85 \%$ de los casos de TB pulmonar con baciloscopia positiva; este indicador mostró un incremento del 22 al $68 \%$ entre 2001 y 2008, pero se deterioró en 2010 (Figura1).

\section{IMPACTO EPIDEMIOLÓGICO DE LA POLITICA}

En Colombia, el control de la TB ha dado lugar a un importante debate político, por cuanto la TB no solamente es un trazador del desempeño del sistema de salud sino un indicador de desarrollo social ${ }^{(25)}$. La norma técnica (resolución 412) de control de la TB, define los indicadores que se pretenden lograr en 2015. Entre ellos, una tasa de incidencia (TI) de 16 por 100 000 habitantes y una tasa de mortalidad (TM) de 1,8 por 100000 habitantes, con respecto a 1993, cuando la TI fue 31,98 por 100000 habitantes y la TM de 3,6 por 100000 habitantes. Estas metas no se han logrado a la fecha; en 2010 y para el 2012, la TI estuvo en 34 casos por 100000 habitantes.

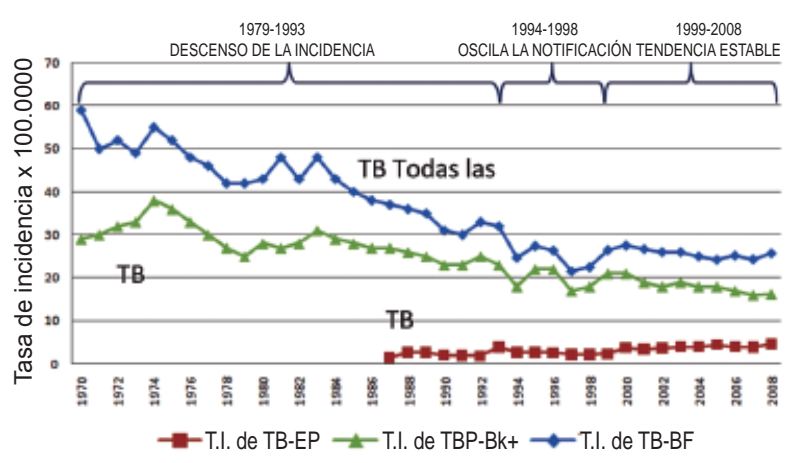

Figura 2. Incidencia de la tuberculosis en Colombia, 1970$2008^{(16)}$

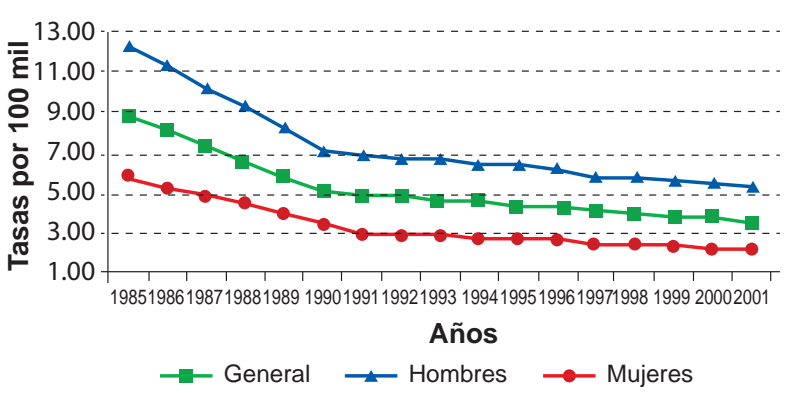

Figura 3. Tendencia de la mortalidad registrada por tuberculosis en Colombia 1985-2001(2).

Según el Ministerio de Salud de Colombia, la incidencia de TB en el período de 1970 al 2008 configura tres etapas (Figura 2). La primera corresponde al programa vertical de control vigente entre 1970 y 1993; durante la segunda fase, de 1994 a 1998, que coincide con la transición del modelo de política y la descentralización del programa, la incidencia muestra una oscilación marcada. La tercera etapa que va de 1999 a 2008, corresponde a la consolidación del modelo; con más estabilidad según los datos de notificación. Para este período se incursionó el Plan de Atención Básica que fortaleció la estrategia en el control de la TB ${ }^{(26)}$. Durante los primeros años de introducida la ley 100 de 1993, la determinación de SR estudiados con baciloscopia en Colombia, permanece estable, alrededor de 3 a 6 casos por 1000 habitantes (16), el indicador decae posteriormente sugiriendo un deterioro en la detección y notificación de los SR. Otros autores consideran que esta gráfica refleja más el deterioro en la búsqueda de casos que un impacto real sobre la enfermedad (3).

El análisis de la mortalidad por TB registrada oficialmente muestra una disminución de las defunciones entre 1985 y 1990, pero marca una desaceleración a partir de 1991 cuando se desmonta el SNS y se implantan las reformas (Figura 3)

\section{CONCLUSIONES}

La información analizada sugiere que la TB ha persistido en Colombia como problema prioritario de salud pública. Durante la década de los 80 se observó un esfuerzo en la gestión de los programas de control que se reflejó en la reducción de los casos nuevos registrados y en la mortalidad. A partir de 1993, cuando se desmontó el Sistema Nacional de Salud y se privatizó la gestión de los recursos, los indicadores de desempeño del programa de control mostraron un 
deterioro importante que se refleja particularmente en la mortalidad; es posible también que hayan aumentado la incidencia y los casos resistentes, pero la información disponible sobre estos eventos presenta serias limitaciones.

El deterioro en el control de la TB se ha atribuido a fallas estructurales en el modelo de gestión de la salud adoptado por Colombia. A partir de 2009 se observa un esfuerzo por precisar y fortalecer la política de control; estas políticas, sin embargo, no tocan la estructura del modelo de salud y los resultados encontrados no mues- tran aún una recuperación importante. En este contexto puede ser difícil que Colombia logre las metas de control de TB previstas a 2015.

Agradecimientos: a la universidad de Cartagena. Programa Doctorado en medicina tropical. A la estrategia de Sostenibilidad de la Facultad Nacional de Salud Pública, Universidad de Antioquia 2013-2014.

Fuentes de financiación: autofinanciado.

Conflictos de interés: los autores declaran no tener conflictos de interés.

\section{REFERENCIAS BIBLIOGRÁFICAS}

1. McKeown T. El papel de la medicina: sueño, espejismo o némesis? México: siglo XXI; 1982

2. Gómez-Arias RD; Universidad de Antioquía. La mortalidad evitable como indicador de desempeño de la política sanitaria Colombia 1985-2001. Medellín: Editorial Universidad de Antioquia; 2008.

3. Ayala C, Kroeger A. La reforma del sector salud en Colombia y sus efectos en los programas de control de tuberculosis e inmunización. Cad Saude Publica. 2002;18(6):1771-81

4. De Groote T, De Paepe P, Unger JP. Colombia: prueba in vivo de la privatización del sector salud en países en desarrollo. Rev Fac Nac Salud Publica. 2007;25(1):106-17.

5. Yepes F, Sánchez L. La reforma del sector de salud en Colombia: ¿un modelo de competencia regulada? Rev Panam Salud Pública. 2000;8(1-2):34-41.

6. Arbeláez MP. La reforma del sector salud y el control de la tuberculosis en Colombia. En: Yadón Z, Gürtler R, Tobar F, Medici A, editores; Organización Panamericana de la Salud. Descentralización y gestión del control de las enfermedades transmisibles en América Latina [Internet]. Buenos Aires: OPS; 2006. p. 115-29 [citado el 23 de junio de 2012]. Disponible en: http://cidbimena.desastres.hn/filemgmt/files/resdescentralizacion.pdf

7. Arbeláez MP, Gaviria MB, Franco A, Restrepo R, Hincapié D, Blas E. Control de la tuberculosis y competencia regulada en Colombia. Int J Health Plann Mgmt. 2004;19:S25-S43.

8. Gómez R. Efectos de la ley 100 sobre la institucionalidad de la salud en Colombia. Seminario internacional "El derecho a la salud en Colombia y la reforma al sistema de salud", 9 y 10 de marzo de 2005 [Internet]. Medellín: Facultad Nacional de Salud Pública; 2005 [citado el 3 de agosto de 2013]. Disponible en: http:// guajiros.udea.edu.co/fnsp/cvsp/efectos\%20de\%20la\%20ley\%20100.pdf

9. Varela A, Carrasquilla G, Tono T, Samper B. Asimetría en la información: Barreras para la implementación de la reforma en Colombia. Colomb Med. 2002;33(3):95-101.

10. Cardona A, Mejía LM, Nieto E, Restrepo R. Temas críticos en la reforma de la Ley de seguridad social de Colombia en el capítulo de salud. Rev Fac Nac Salud Pública. 2005;23(1):117-33.

11. Carvajal R, Cabrera G, Mosquera J. Percepciones de los efectos de la implementación del Sistema General de Seguridad Social en Salud sobre las acciones de control de tuberculosis en el Valle del Cauca, Colombia. Colom Med. 2004;35(4):179-184

12. Málaga $\mathrm{H}$, Latorre MC, Cárdenas J, Montiel H, Sampson C, Taborda $\mathrm{M}$, et al. Equidad y reforma en salud en Colombia. Rev Salud Publica. 2000;2(3):193-219.

13. Victoria J. El control de la tuberculosis en el sistema de general de seguridad social en salud de Colombia. Medicas UIS. 1998;12:265-9.

14. Estos son los nuevos retos del Plan Obligatorio de Salud [Internet]. Bogotá: El Tiempo; c2013 [citado el 1 de Julio de 2012]. Disponible en: http:// www.eltiempo.com/archivo/documento/CMS-11990418

15. Colombia, Ministerio de la Protección Social. Resolución 412 de 2000. Tomo 2, pág.65. 25 de febrero del 2000.
16. Colombia, Ministerio de la Protección Social, Instituto Nacional de Salud, Organización Panamericana de la Salud. Plan estratégico. Colombia libre de tuberculosis 2010-2015. Para la expansión y fortalecimiento de la estrategia Alto a la TB. 3ra ed. Bogotá: MPSINS: 2009.

17. Organización Mundial de la Salud. Estadísticas sanitarias mundiales 2011. En: Mortalidad y morbilidad por causas específicas. p. 57-78. OMS: Ginebra; 2011.

18. El Banco Mundial. Informe indicadores. Incidencia de tuberculosis [Internet]. Ginebra: OMS; 2012 [citado el 23 de marzo de 2013]. Disponible en : http://datos.bancomundial.org/indicador/SH.TBS.INCD

19. Organización Panamericana de la Salud. Informe PAHO Colombia Tuberculosis [Internet]. Bogotá: OPS; 2011 [citado el 20 abril de 2012]. Disponible en: http://new.paho.org/col/index. php ?option $=$ com_content $\&$ task $=$ vie w\&id=1028\&Itemid $=1$

20. Malbrán Carlos. Tuberculosis Multirresistente. Lima: INER; 2011.

21. Caminero JA. Origen, presente y futuro de las resistencias en tuberculosis. Arch Bronconeumol. 2001;37:35-42.

22. Instituto Nacional de Salud. Lineamientos para el manejo programático de pacientes con tuberculosis fármacoresistente [Internet]. Bogotá: INS; 2013 [citado el 16 de marzo de 2012]. Disponible en: http://www.asoneumocito.org/wp-content/uploads/2014/08/ Lineamientos-manejo-de-Tuberculosis-Farmacorresistente.pdf

23. Organización Mundial de la Salud. DOTS-Plus \& y el Comité Luz Verde (Green Light Committee) [Internet]. 
Ginebra: OMS; 2001 [citado el 16 de marzo de 2013]. Disponible en: http:// whqlibdoc.who.int/hq/2000/WHO_ CDS_TB_2000.283_spa.pdf

24. Colombia, Ministerio de la Protección Social. 24 de Marzo Día Mundial de la Tuberculosis. Vivamos sin tuberculosis. Todos somos parte de la solución [Internet]. Bogotá: MPS; 2011 [citado el 22 de junio de 2012]. Disponible en: https://www.google.com.pe/url? $\mathrm{sa}=\mathrm{t} \& \mathrm{rct}=\mathrm{j} \& \mathrm{q}=\& \operatorname{esrc}=\mathrm{s} \&$ source $=\mathrm{w}$ eb $\& \mathrm{~cd}=6 \& \mathrm{cad}=\mathrm{rja} \&$ uact $=8 \& \mathrm{ved}=$ 0CCoQFjAF\&url=http\%3A\%2F\%
2Fwww.paho.org\%2Fcol\%2Findex. ph p \% 3 Foption \% 3 D com docman\%26task\%3Ddoc_download\% 26gid\%3D880\%26Itemid\%3D\&ei=R qRvVJLGNoSYNuK7hLAN\&usg=AF QjCNHnPZ4fFGnpogiOjWlO9HpL vzOOKQ\&sig2=_j3CElcz9HO8oCA CtStyWA\&bvm=bv.80185997,d.eXY

25. González A, Mogollón O. La reforma de salud y su componente político: Un análisis de factibilidad. Gac Sanit. 2002 Ene-Feb;16(1):39-47.

26. Castiblanco CA, Llerena C. Tuberculosis en Colombia: Análisis de la situación epidemiológica, año 2006. Asociación Colombiana de Infectología. 2008;12(3):159-73.

Correspondencia: Dilia Fontalvo Rivera Dirección: Facultad de Medicina Universidad de Cartagena. Barrio Zaragocilla. Avenida del Consulado. Calle 30 Número 48-152. Cartagena de Indias, Colombia. Teléfono: 3114002763

Correoelectrónico:diliafontalvor@gmail.com

\section{Descarge de forma gratuita los contenidos de la Revista Peruana de Medicina Experimental y Salud Pública, ingrese a WWW.ins.gob.pe, seleccionando el ícono de la revista.}

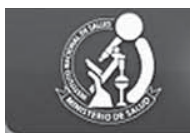

\section{MINISTERIO DESALUD}

INSTITUTO NACIONAL DE SALUD

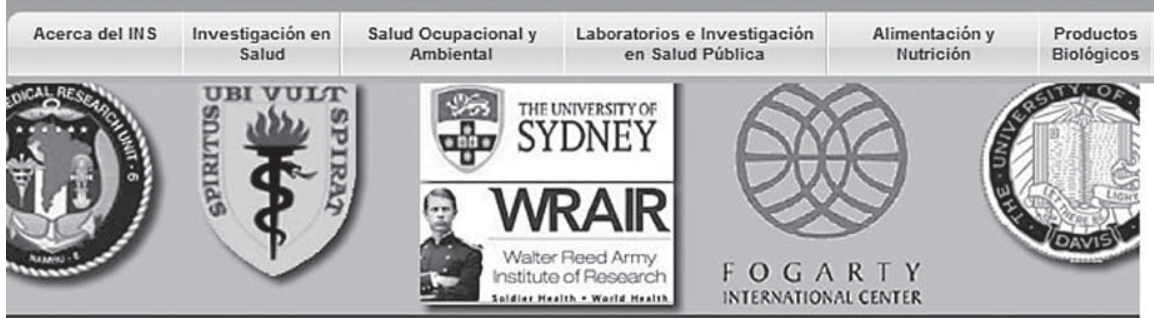

Phylogenetics and Bioinformatics Sequence Analysis Training, to be held January 5-9,2015 at Universidad Peruana Cayetano Heredia, in Lima, Peru.

BOLETIN INSTITUCIONAL Y ÚlTIMAS INVESTIGACIONES Ver TOdas

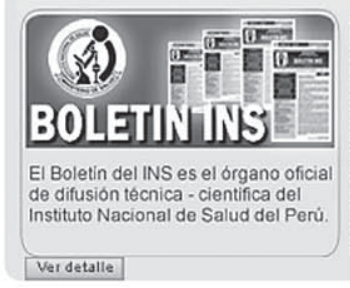

CALENDARIO DE EVENTOS

- Noviembre 2014 . Do Lu Ma Mi Ju Vi Sa \begin{tabular}{l|l|l|l|l|l|l|l|}
2 & 3 & 4 & 5 & -6 & 7 & -8
\end{tabular} \begin{tabular}{l|l|l|l|l|l|l|}
9 & 10 & 11 & 12 & 13 & 14 & 15
\end{tabular} $\begin{array}{llllllll}16 & 17 & 18 & 19 & 20 & 21 & 22\end{array}$ \begin{tabular}{l|l|l|l|l|l|l}
23 & 24 & 25 & 26 & 27 & 28 & 29
\end{tabular}

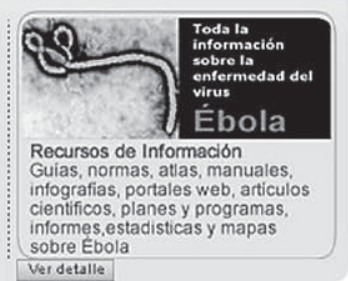

NOTIIAS

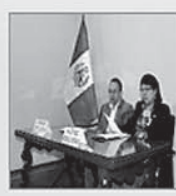
de edad

25/11/2014

Ver detalle,
GALERÍA

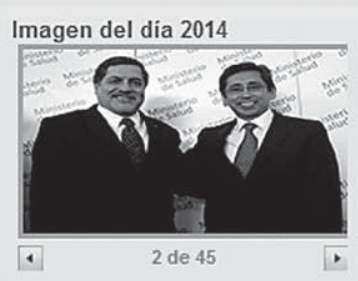
CURSOS YEVENTOS PUBLICACIONES

PRODUCTOS Y SERVICIOS

Anemia y Desnutrición en niños menores de un año

El último Viernes Cientifico del 2014 logró reunió a 220 profesionales, técnicos y estudiantes interesados en la problemática de la anemia y desnutrición en niños menores de un año de edad, en el auditorio de la sede central del Instituto Nacional de Salud.
Buscar..

Control de Calidad Salud
Intercultural
de Medicamentos

Q Portal del Transparencia

Mestión de calidad

6 Directorio Telefónico

Enlaces de Interes

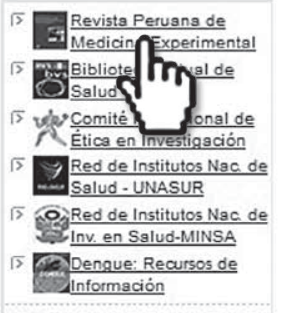

Ver más

Servicios Web

NEUढ@ TRAMITE DOCUMENTARIO

PAGOA PROVEEDORES

I5 La Mejor Receta

$\sqrt{5}$ Libro de Redamaciones

5 Ensayos dínicos

$\sqrt{5}$ Reas-Net

(15 Directorio de investigadores

$\sqrt{5}$ Fichas Net

(5) Convocatoria de personal

$\sqrt{5}$ Financiamiento Externo: Convocatorias vigentes 